\title{
Flat, Broadband Supercontinuum Generation at Low Pulse Energies in a Dispersion-Tailored Lead-Silicate Fibre
}

\author{
Giorgio M. Ponzo*, Xian Feng, Peter Horak, Francesco Poletti, Marco N. Petrovich, Wei H. Loh \\ and David J. Richardson \\ Optoelectronics Research Centre, University of Southampton, Southampton, SO17 1BJ, UK \\ *Corresponding author: gmplg09@orc.soton.ac.uk
}

\begin{abstract}
We report flat, broadband supercontinuum generation at low pump pulse energies (69pJ $740 \mathrm{fs}$ pulses at $1540 \mathrm{~nm})$ in a lead-silicate fibre providing a unique combination of high nonlinearity $\left(820 \mathrm{~W}^{-1} \mathrm{~km}^{-1}\right)$ and a flat near-zero dispersion profile.
\end{abstract}

OCIS codes: (320.6629) Supercontinuum generation; (060.4370) Nonlinear optics, fibers;

\section{Introduction}

Flat supercontinua (SC) generated with low power pump lasers are of interest in various applications, including amongst others the realization of compact sources for wavelength division multiplexing (WDM) [1] and optical coherence tomography [2]. A significant spectral broadening with low input powers can be produced by using fibers with high effective nonlinearity, i.e. fabricated with high nonlinear index $\left(\mathrm{n}_{2}\right)$ glasses and small effective mode areas. To this end, several compound glass systems are suitable materials, since they can have a substantially higher nonlinear index as compared to silica. The dispersion profile is also paramount both to reduce the input power requirements and to control the shape of the SC generated, since it strongly influences the type and relative gain of nonlinear mechanisms responsible for the broadening. For instance, fibres with convex and near-zero dispersion profiles can produce flat and broad SC [3]. Tailored dispersion profiles can be achieved with the holey-fibre (HF) technology, by designing the hole diameter and the pitch of a cladding comprising multiple rings of holes [3]. However, while multi-ring holey fibre designs are well-established in silica, they are difficult to achieve using a compound glass; indeed, due the low softening point and lower thermal stability, the realisation of complex structures with multiple rings is difficult using compound glass and in particular the control of structural parameters to the accuracy required for dispersion control (a few hundred nm typically) is extremely challenging [4]. The situation is even worse for fibres with near-zero dispersion profiles, which require more accurate structural control, due to the low dispersion values targeted near the maximum. This ultimately makes it difficult to simultaneously achieve the highest possible nonlinearity values and a tailored dispersion profile.

Despite this issue, by combining an all-solid approach with a simple geometry, we recently managed to accurately control the fibre structure and fabricate the first non-silica fibre with an ultra-flat, near-zero dispersion profile [5]. This fibre also allowed us to obtain maximum nonlinearity, a tailored dispersion profile, and single mode guidance simultaneously, which is not feasible using conventional HF designs. Due to the W-type index profile, we refer to this fibre as W-fibre, for conciseness.

In this work we report SC generation experiments for a W-fibre with two close zero dispersion wavelengths centered near $1550 \mathrm{~nm}$ and a maximum anomalous dispersion value of $+2 \mathrm{ps} / \mathrm{nm} / \mathrm{km}$. We also report numerical simulations and a discussion of the nonlinear mechanism responsible for the flat and uniform broadening.

\section{Supercontinuum generation experiments}

For the fabrication of the lead-silicate W-fibre we employed a techique described in detail in [5]. A combination of different glasses from the same lead-silicate family was used: the fibre core is made up of SF57 glass, and the cladding of LLF1 glass (from Schott). This simple structure is sufficient to achieve high nonlinearity and to tailor the dispersion profile [4]. Indeed, the high nonlinear index of the SF57 glass and the small mode area results in a very high effective nonlinearity $\left(820 \mathrm{~W}^{-1} \mathrm{~km}^{-1}\right)$ [5]. On the other hand, the index difference between the SF57 core and LLF1 cladding glasses, and the chosen core diameter provide a convex near-zero dispersion profile with the maximum near $1550 \mathrm{~nm}$. In order to ensure single mode guidance through the suppression of weakly guided higher order modes, a second, outer cladding (SF6 glass), with an intermediate refractive index between LLF1 and SF57, was added within the fibre structure, resulting a W-shaped refractive index profile. Fibre fabrication presents numerous challenges due to the substantially different viscosities of the three glasses; however, by using a rod-in- 
tube-method we managed to achieve high quality structures (a scanning electron microscope (SEM) image is shown in the inset of Fig. 1), with losses very close to the bulk loss of SF57 glass. The present work is based on an improved fibre, which, in addition of high nonlinearity, achieves a flat dispersion profile with two closely spaced zeros. The fibre had a core of $1.66 \mu \mathrm{m}$ and a transmission loss of about $2 \mathrm{~dB} / \mathrm{m}$ at $1550 \mathrm{~nm}$. Numerical simulations of the overall propagation loss spectrum (Fig. 1) revealed a rapid increase beyond $2 \mu \mathrm{m}$, due to the poor modal confinement at these wavelengths, resulting from the small core diameter. The fibre dispersion profile over a $400 \mathrm{~nm}$ wide wavelength interval was accurately measured through white light interferometry using a commercial SC source (Fig. 1). Our fibre has a flattened dispersion with variation limited to $5 \mathrm{ps} / \mathrm{nm} / \mathrm{km}$ over $200 \mathrm{~nm}$, two zero dispersion wavelengths (ZDWs) separated by about $100 \mathrm{~nm}$, and a small maximum anomalous dispersion value of $+2 \mathrm{ps} / \mathrm{nm} / \mathrm{km}$. The simulated dispersion profile agrees reasonably well with the experimental data (Fig. 1); we speculate that the very small deviation may be due to differences between theoretical and actual refractive index profile caused e.g. by ion diffusion during fibre drawing or residual stress.
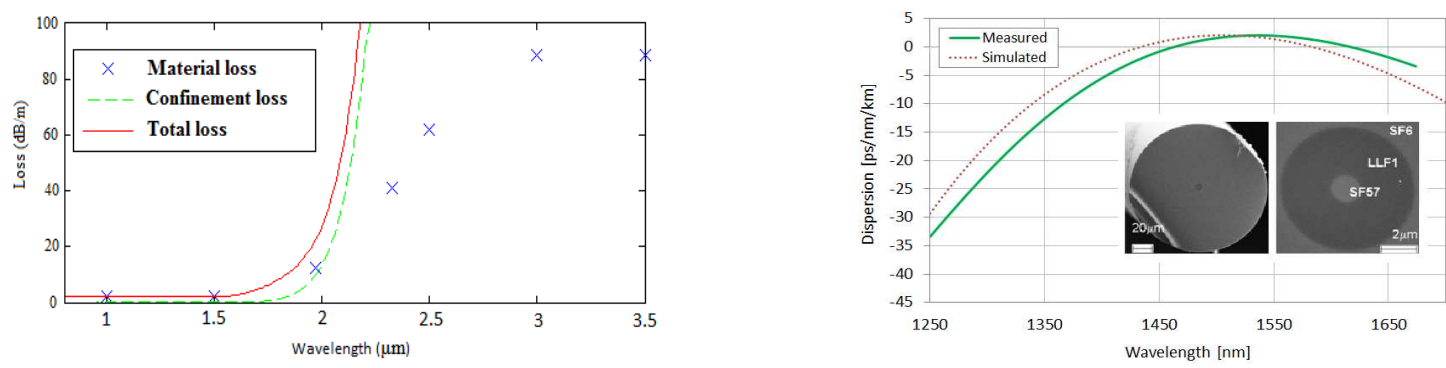

Fig. 1. Left: calculated loss spectrum. Right: measured and calculated dispersion profiles and scanning electron microscope (SEM) image of the W-fibre (inset)

For the SC generation we used a compact fibre laser emitting linearly polarized, 774fs long pulses at $1540 \mathrm{~nm}$ wavelength, which falls very conveniently in between the two ZDWs of our fibre. The repetition rate was $40 \mathrm{MHz}$ and the average output power was $60 \mathrm{~mW}$, which could be regulated using a variable neutral density filter. Due to the slight birefringence, a half-wave plate was used to evaluate the effect of using different input polarization planes on the SC generated. The input pulses were coupled into a $1.2 \mathrm{~m}$ long fibre sample using an antireflection coated $60 \mathrm{x}$ objective. The output light was butt-coupled to a single mode fiber (SMF) and measured with an optical spectrum analyser (OSA).
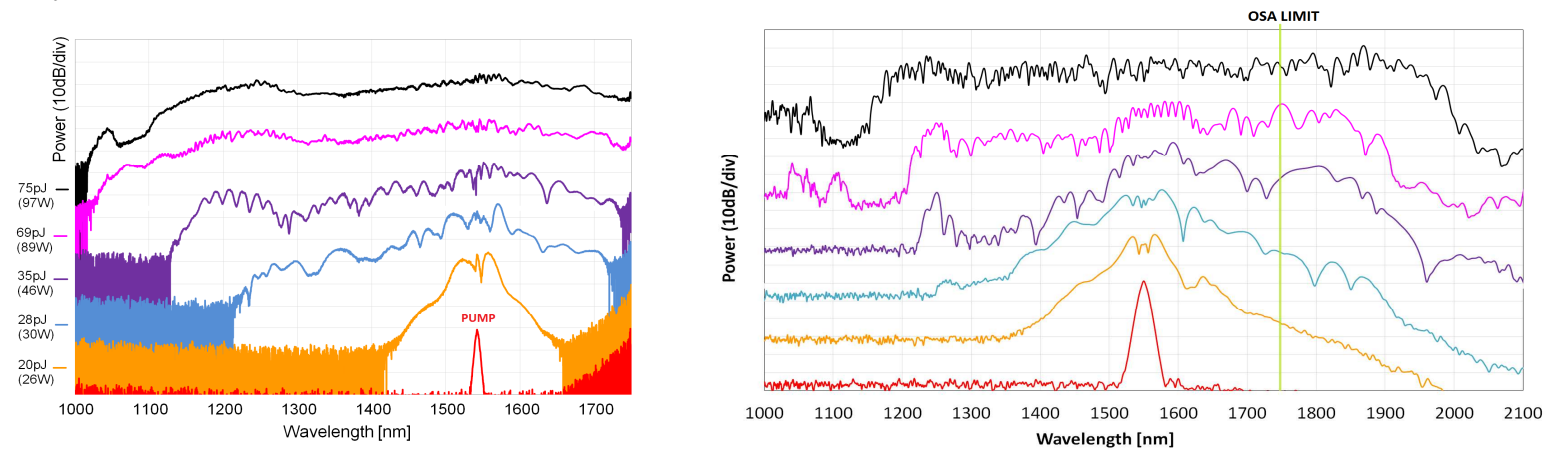

Fig. 2. Experimental (left) and calculated (right) SC spectrum evolution with input power. The traces have been vertically offset by $20 \mathrm{~dB}$ for clarity

After careful optimization of the coupled power a launch efficiency of $20 \%$ was obtained. The input polarization influenced the amount of noise and ripples in the measured SC spectrum, which could be minimized for particular positions of the half-wave plate, corresponding to the fibre's axes. Fig. 3 shows the output spectra recorded at different pulse energies. Due to the wavelength range of the OSA the long wavelength edge of the spectral measurement was limited to $1750 \mathrm{~nm}$. A maximum broadening was already achieved for a pulse energy of $69 \mathrm{pJ}$, corresponding to a peak power of $89 \mathrm{~W}$ which is one of the lowest pump pulse energies ever reported for femtosecond pumping achieving such a broad spectrum, in a non-tapered fibre. For comparison, in [6] an octave spanning SC was generated in an SF6 photonic crystal fibre with 200pJ, 60fs pulses, corresponding to a peak power of $3 \mathrm{KW}$. In [7] 20pJ was sufficient to obtain a broad SC. However, the corresponding peak power was 300W (60fs pulses). In [8] a SC was generated at telecom wavelengths with 200pJ and 60fs pulses. 
From Fig. 2 it is seen that a SC with flatness of about $5 \mathrm{~dB}$ was achieved over the entire bandwidth (including any residual pump) and a highly symmetric spectrum is maintained for each pump power level. While our measurements were limited to $1750 \mathrm{~nm}$ due to the range of the OSA, the high degree of symmetry observed suggested that the SC spectrum extending to approximately $2 \mu \mathrm{m}$ for the highest values of pump power employed. In order to provide insight into the spectrum at longer wavelengths, we performed numerical simulations of the corresponding generalized nonlinear Schrödinger equation using a standard split-step Fourier tool, which takes into account the full dispersion profile as well as nonlinear Kerr and Raman effects. The dispersion profiles in Fig. 1, the measured nonlinear coefficient $\left(820 \mathrm{~W}^{-1} \mathrm{~km}^{-1}\right)$ and the calculated loss spectrum in Fig. 1 were used as inputs to these simulations. Fig. 2 shows the resulting SC spectra for different input power levels, which confirmed qualitatively the experimental spectral evolution and the symmetric broadening around the pump for all input power levels investigated. As expected, the generated SC was limited to $2 \mu \mathrm{m}$ at long wavelengths. Numerical simulations indicated that the long wavelength limit is due to the substantial increase of the propagation loss beyond $2 \mu \mathrm{m}$ and which also precludes further broadening at the short wavelength edge. Therefore, a broader SC cannot be generated in this fibre with this pump configuration.

At low powers, the SC is characterized by a self-phase modulation process which causes a uniform and symmetric spectral broadening around the pump. A high degree of coherence is preserved over the full spectrum in this case. The uniform broadening is then broken at increasing power levels by four wave mixing (FWM) peaks emerging from the noise level. Evidence of the FWM process is visible both in the experimental and the simulated spectra (Fig. 2). At higher input powers, the two FWM peaks merge with the spectrum and a uniform and flat SC is generated. The nonlinear mechanisms identified agree with those reported in [3] for a silica based fibre with a similar flat dispersion profile. Simulations also indicate that, when the two peaks merge the coherence of the SC drops dramatically across the full spectrum. A low coherence SC is desirable in some OCT applications, where the resolution is related to the coherence length of the source [2]. If needed, according to [9], a high degree of coherence could be achieved over the entire SC spectrum using shorter pulses (50fs) and an all-normal dispersion profile with maximum value smaller than about $-10 \mathrm{ps} / \mathrm{nm} / \mathrm{km}$ [10] are required (e.g. the dispersion curve of Fig. 1 shifted slightly towards the normal dispersion region).

\section{Conclusions}

We have demonstrated SC generation in a lead-silicate fibre with a tailored dispersion profile. The ultra-flattened near zero dispersion profile allowed us to obtain a broad and flat (flatness of 5dB) SC at telecom wavelengths (centered at $1540 \mathrm{~nm}$ ). The unique design, which allows both a tailored dispersion profile and high nonlinearity $\left(820 \mathrm{~W}^{-1} \mathrm{Km}^{-1}\right)$, led to a SC generated with very low input peak powers of $89 \mathrm{~W}$ corresponding to $69 \mathrm{pJ}$ pulse energy. Numerical simulations qualitatively confirmed the SC spectrum evolution with input power and revealed the nonlinear mechanisms responsible for the broadening to be the combination of self-phase modulation (SPM) and four wave mixing (FWM). The relatively long pulses (774fs) used along with the low pulse energies required is promising for the realization of compact sources of flat SC for telecom and optical coherence tomography applications.

\section{References}

[1] Y.Takushima, K. Kikuchi, “10-GHz, Over 20-Channel Multiwavelength Pulse Source by Slicing Super-Continuum Spectrum Generated in Normal-Dispersion Fiber”, IEEE Photon. Technol. Lett. 11, 322-324 (1999).

[2] A Unterhuber et al, "Advances in broad bandwidth light sources for ultrahigh resolution optical coherence tomography", Phys. Med. Biol. 49, 1235 (2004).

[3] V. Tse et al., "Supercontinuum generation at $1.06 \mu \mathrm{m}$ in holey fibers with dispersion flattened profiles," Opt. Express 14, 4445-4451 (2006)

[4] X. Feng et al., "Dispersion controlled highly nonlinear fibers for all optical processing at telecoms wavelengths," Opt. Fiber Tech. 16, 378$391(2010)$

[5] A. Camerlingo et al., "Near-zero dispersion, highly nonlinear lead-silicate W-type fiber for applications at 1.55 $\mu$ m," Optics Express 18, 15747-15756 (2010).

[6] H. Hundertmark et al., "Octave-spanning supercontinuum generated in SF6-glass PCF by a 1060 nm mode-locked fibre laser delivering 20 pJ per pulse," Opt. Express 17, 1919-1924 (2009).

[7] H. Hundertmark et al. , "Supercontinuum generation with $200 \mathrm{pJ}$ laser pulses in an extruded SF6 fiber at $1560 \mathrm{~nm}$," Opt. Express 11 , $3196-$ 3201 (2003).

[8] G. Qin et al, "Zero-dispersion-wavelength-decreasing tellurite microstructured fiber for wide and flattened supercontinuum generation," Opt. Lett. 35, 136-138 (2010).

[9] A. M. Heidt, "Pulse preserving flat-top supercontinuum generation in all-normal dispersion photonic crystal fibers," J. Opt. Soc. Am. B 27, 550-559 (2010). 